\title{
Relaciones Canadiense-Latinoamericanas pasadas, presentes y futuras
}

\begin{abstract}
J. C. M. O C E L S B Y, profesor canadiense, educado en la universidad de Stanford $y$ la School of Oriental and African Studies de la univcrsidad de Londres, es un especialista en las relaciones entre las potencias coloniales en América. Enscũa historia de América Latina en la universidad de Western Ontario, que es uno de los centros de estudios latinoamericanos en Canadá. Anteriormente era profesor de la historia del imperio británico en la universidad de Victoria. Ha publicado más de cincuenta artículos y reseñas en revistas cn, Canadá, los Estados Unidos, Inglaterra y el Caribe. En 1971 recibió una beca del gobierno de Canadá para terminar su estudio sobre las relaciones canadiense-latinoamericanas, 1867-1967.
\end{abstract}

Politica Exterior para Canadienses ${ }^{1}$, el informe final del examen de las relaciones exteriores del Canadá por el gobierno del Primer Ministro Pierre Elliot Trudeau, dedica una sección a América Latina. Es casi como si nunca hubieran tenido relaciones con esa área, ya que el enfoque del trabajo, que subraya la necesidad de fomentar el intercambio, desarrollo, inversiones, de establecer contactos personales más estrechos e intercambio de ideas, parece ser muy novedoso para los canadienses. Ahora, debo admitir que mis investigaciones acerca de las relaciones canadiense-latinoamericanas aparecen no sólo novedosas sino que hasta quijotescas a mis colegas, quienes hán permanecido en el campo más sólido de los estudios canadienseimperiales, especialmente cuando una figura tan prominente de los Estados Unidos como Mr. McGeorge Bundy, ha tildado a los estudios latinoamericanos como un "campo de segunda categoria que atrae a mentes secundarias"2.

*Esta es una revisión de un trabajo presentado ante una reunión auspiciada por la Asociación Canadiense de Estudios sobre América Latina, El Colegio de México y la Universidad Nacional Autónoma de México en mayo de 1971.

'Foreign Policy for Canadians, Latin America (Ottawa, 1970). Hay una versión castellana.

"Citado en John Mander, The Unrevolutionary Sociely, The Power of Latin .American Conservatism in a Changing World (New York, 1969), x-xi. 
No me siento suficientemente competente como para desafiar a un hombre que encuentra otras regiones del mundo, tales como el sudeste de Asia, más dignas de los esfuerzos de mentes de primera categoría, pero lo que estoy en el proceso de descubrir concerniente a las relaciones canadiense-latinoamericanas es estímulo. suficiente para mí. Se pondrá en evidencia que este trabajo no es un estudio completo de estas relaciones, pues todavía queda: mucho por hacer. Por lo tanto, yo propongo presentar unas viñetas de estas relaciones pasadas que espero iluminarán algunas de: las posiciones tomadas en el informe sobre América Latina del: Gobierno canadiense.

El intercambio comercial es una de las áreas de mayor concentración en la Politica Exterior para Ganadienses. Se ha censuradoesta concentración ${ }^{3}$; pero yo creo que el énfasis del Gobierno es típico de una nación que necesita comerciar para sobrevivir. Ac tualmente se ha expresado mucha preocupación respecto al exceso de nexos mercantiles con los Estados Unidos, y no cabe duda que la diversificación es una necesidad. Esto constituia un problema ya en 1865, cuando los representantes de cuatro colonias británicas de Norteamérica (Canadá, Nueva Escocia, Nueva Brunswick y la Isla del Príncipe Eduardo) se reunieron en Quebec aquel mes de septiembre "... en un momento en que nuestro importante comercio con los Estados Unidos se ve amenazado de interrupción, y que de ahora en adelante habrá de continuarse indudablemente bajo normas diferentes a las hasta aquí imperantes." 4 La amenaza norteamericana de abrogar el Tratado de Reciprocidad entre las colonias y su vecina del sur hizo que los representantes de la Norteamérica Británica procurasen estrechar lazos comerciales con el Caribe, Brasil y México. Por consiguiente, solicitaron del gobierno de su Majestad autorización para establecer contactos con dichas regiones, según lo expresaron, para ampliar el comercio "sobre bases más ventajosas." Naturalmente, los miembros de" lo que se llamaba Consejo Confederado para el Comercio decidieron - enviar una comisión a las regiones mencionadas. $Y$ a principios

"Ver por ejemplo algunos de los comentarios en "Forcign Policy for Canadians: Comments on the White Paper", Behind the Headlines (una publicacion del Instituto Canadiense de las Relaciones Internacionales), XXXIX (August, $1970)$.

${ }^{4}$ Report of the Commissioners from British North America appointed to Inquire into the trade of the W'est Indies, Mrexico, Brazil (Ottawa, G. E. Desbarats, 1866), 4.

${ }^{5}$ La primera vez que hallé correspontencia relativa a esta misión fuc en Public Archives of Canada (cn lo sucesivo citado como PAC), Record Group (RG) 7, G 21. Mi colega W. B. Hamilton habia dado con documentos sobre la misión propucsta, mientras investigaba sobre uno de los líderes de Nueva Escocia que 
de 1866 viajó hacia el sur la primera de las que habrian de ser varias misiones canadienses (1941, 1945, 1953, 1968). Muy.complacidos por la acogida que se les dispensó, la cual incluyó una entrevista con don Pedro II, emperador del Brasil, los comisionados, "en vista de que México era aún teatro de guerra, consideraron inoportuno diferir su regreso por una visita a dicho imperio" pero en los demás lugares “... hallaron tanto a los gobiernos como al pueblo ansiosos por obtener informes y por coadyuvar a los propósitos de la misión.". (Esto podría ser un paráfrasis del "Informe Preliminar de la Misión Ministerial a América Latina... 1968")7. Poco se realizó después del regreso de la misión, pues los temores de perder el mercado estadounidense nunca se concretaron; y la política local y el movimiento federalista eran de importancia más inmediata. $s$

Cincuenta años más tarde (1916), cuando el Ministro de Relaciones Exteriores brasileño, Dr. Lauro Muller, visitó Washington, Bonar Law, el Ministro de Colonias británico nacido en el Canadá, sugirió que Canadá lo invitara a realizar una visita. Lauro Muller no hablaba inglés, y con su francés "se sentiría más a gusto en el Canadá que en los Estados Unidos"0. Muller vino, le agradó la atención recibida, y pareció que nacía una nueva era en las relaciones comerciales entre Canadá y Brasil. La revista comercial brasileña Ferrocarril comentó que "si los canadienses saben poco acerca del Brasil nosotros sabemos aún menos de cuestiones canadienses y por esta razón la importancia de este suceso ha pasado desapercibida... Canadá consume una considerable cantidad de productos tropicales importados de este pais por los Estados Unidos, y sin duda Brasil importa muchos artículos canadienses importados por los Estados Unidos"10. En 1945, el señor J. A. Mac Kinnon, Ministro de Intercambio y Comercio, y antiguo jefe de las misiones que en 1941 y 1945 habían visitado la América Latina declaró en la Cámara de los Comunes: "Esperamos pronto poder restablecer rutas de embarque directas a los países latinoamericanos, en vez de tener que enviar nuestra mercadería por intermedio de los Estados Unidos, lo cual deberá incrementar nues-

habia participado en la misión. Estoy en deuda de gratitud hacia él por haberme proporcionado la fuente de la cita correspondiente: J. Castell Hopkins (cd.), Canada, An Encyclopedia of the country... (Toronto, 1900), 300.

"Report of the Commissioners, vii.

"Hopkins, Canada, 300. Ver también "Prcliminary Report", 7.

"Hopkins, Canada, 300.

${ }^{\circ}$ Bonar Law a Goberriador General, 13 de julio de 1916, PAC, RG 7, G 21/19040.

${ }^{10}$ Citado en Peel a Grey, 14 de setiembre de 1916, PAG. RG 7, G 21/19040. 
tro comercio con los países latinoamericanos de una manera substancial"11. ¿Dónde hemos leído esto antes? Pues, lo referiré a la página 31 de Polilica Exterior para Canadienses, América Latina. Dice:

Entre los problemas generales que afectan el comercio entre Canadá y América Latina está el de fomentar un intercambio más directo en ambas direcciones. Tanto en el caso de exportación canadiense a América Latina como en el de exportación latinoamericana al Canadá, una alta proporción de este intercambio se realiza por intermedio de los Estados Unidos. Parecería haber buen fundamento para examinar la posibilidad de transporte aéreo y maritimo más directo, especialmente con respecto a embarques aéreos de mercancia delicada de estación de rápido deterioro, en vista de que las estaciones de este país y de la mayor parte de América Latina son complementarias.

¿Hasta dónde han progresado las actitudes canadienses en estos ciento tres años? ${ }^{22}$

${ }^{11}$ Canadian House of Commons Debales, LxxXry, N9 70 (December 11, $1945), 3371$.

12Durante todos cllos se repite una nota familiar. Si el actual gobierno del scñor Trudeau hubiera estado enterado de lo que anteriormente se había dicho, quizá hubiera sido más original. Tal como es, la actual declaración tiene su equivalente en el informe de la Comisón de 1866. Dicho informe destacaba que el escaso comercio entre Brasil y la Nortcamérica Británica no reflejaba el comercio realizado por vía de Inglaterra o los Estados Unidos, el cual era "sin duda mucho major, y es de lamentar que... [dicho comercio] no pueda determinarse con exactitud." Tampoco se logró determinar el comercio con Cuba "el cual... ya es muy considerable, aunque su pleno alcance y valor no aparecen en las estadisticas publicadas... ya que gran parte de este comercio hasta aqui se ha hecho por puertos de los Estados Unidos en tránsito de Cuba a las Provincias Británicas y viceversa."

El A Memorandum to the Under-Secretary of Slate for External Affairs on the Development of Canadian Trade with the Republics of South America de diciembre de 1930 afirmaba que algo podia hacerse para ampliar las relaciones comerciales con esa región, a través de los Estados Unidos. Pero el aumento rlependeria del mejoramiento del transporte $y$ comunicaciones oceánicas. Este memorándum destacaba el continuado problema de la preferencia de los canadienses por los productos anunciados en las revistas de los Estados Unidos. De las 540 toncladas de café importadas por Canadá en 1929, 534 procedian de los Estados Unidos, pais no muy conocido por su producción cafetalera. El informe scñalaba que "cl café de la casa Maxwell probablemente sea mejor conocido por cl ama de casa canatliense, que cualquier marca canadiense competidora, y está dispucsta a pagar unos cuantos centavos más por dicho producto, por el hecho de que "se anuncia en Ladies Home Journal [revista para scñoras]". Ello es igualmente cierto hoy dia respecto a los bananos, el café $y$ las naranjas. Mi propio proveedor de frutas me ha dicho que sus clientes insisten en pedir bananos marca 
Los esfuerzos de los canadienses en el campo de inversiones y desarrollo también son bastante ilustrativos. En realidad, podria decirse que son notables en las primeras décadas de este siglo, pues el mayor ímpetu para la inversión canadiense en América Latina fue proporcionado por el capital sobrante disponible para los principales accionistas del ferrocarril (Canadian Pacific Railway). Sir William Van Horne, William Mackenzie, Donald Mann, James Ross, R. B. Angus, y E. R. Wood, entre otros, tenían dinero para tirar a la hoguera después de 1885 y decidieron invertirlo fuera del Canadá, principalmente en las dos cosas que ellos conocían mejor: la construcción de ferrocarriles y la energía eléctrica. ${ }^{13}$

Las inversiones en ferrocarriles se concentraron en Cuba, Guatemala, México y Brasil. La pericia estadounidense y canadiense se combinó con el dinero canadiense y británico para llevar a cabo estas empresas. Es interesante notar que en la construcción de Ios ferrocarriles se empleó no sólo dinero canadiense, sino también las técnicas del ferrocarril canadiense. En Cuba, Sir William Van Horne trató de persuadir al General Leonard Wood, el procónsul estadounidense, para introducir un programa de registro de tierras y un sistema de planeamiento como el primer paso en la reconstrucción de la agricultura cubana. También instó al gobierno militar estadounidense a que tomara la tierra no cultivada de sus dueños ausentes y la distribuyera en parcelas individuales. Los comandantes estadounidenses no aceptaron estas sugerencias. Pero Van Horne redactó la ley de ferrocarriles cubana que establecía una junta directiva, igual que en el Canadá. Esta ley no agradó a los empresarios, pero Van Horne, demostró con éxito que protegería a todos los intereses, incluyendo a los propios. ${ }^{14}$

El representante de Van Horne en Cuba era un joven americano, Percival Farquhar, quien se convirtió en "titán" en el Brasil, donde una de sus empresas de mayor envergadura fue la construcción de ferrocarriles. Farquhar utilizó la experiencia canadiense al tratar de abrir el sudeste brasileño. Introdujo el sistema de

"Chiquita" y naranjas "Sunkist" aun cuando él ha procurado venderles otras de sabor superior.

No resultará fácil mejorar los contactos mercantiles directos entre Canadá y la América Latina Iuchando con gustos canadienses que reflejan el poder de la propaganda estadounidense $y$ de la influencia estadounidense en la distribución normal de alimentos tropicales y semitropicales en el Canadá. No es de esperar que sc produzcan muchos cambios.

${ }^{15}$ L. C. and F. W. Park, The Anatomy of Big Business (Toronto, 1962), 136.

${ }^{11}$ Walter Vaughan, The Life and Work of Sir William I'an Horne (New York, 1920), 286, 292.293. 
planeamiento de Torrens y un sistema de registro de tierras en esa región, y hasta contrató a un oficial de inmigración del ferrocarril canadiense (C. P. R.) para que desarrollara la inmigración en los campos del Brasil. El ferrocarril quebró y el plan de inmigración fracasó pues el oficial se fue a pelear por su Rey y su Patria en 1915.15

Las empresas de energía eléctrica en Río de Janeiro, São Paulo, la Ciudad de México, Monterrey, y otras partes, eran más grandes. y de mayor duración. Brazilian Traction (ahora Brascan) es el más grande de estos esfuerzos y surgió del contacto entre un empresario estadounidense, F. S. Pearson, y capitalistas canadienses. Aparentemente Pearson no podía obtener capital estadounidense para financiar su plan de comprar la concesión de tranvias de tracción a sangre de São Paulo. Encontró apoyo en Canadá, y en 1899 se formó la compañía de Tracción, Luz y Fuerza de São Paulo en Ontario. Los estadounidenses construyeron la compañía con normas estadounidenses, mientras que los aspectos legales y financieros fueron dirigidos por canadienses y británicos. Uno de estos canadienses fue Alexander Mackenzie, quien llegó a tener mucha influencia en el Brasil entre 1900 y 1930 . Si se quería llevar algo. a cabo, aparentemente era una buena idea ir a ver a Mackenzie, quien sucedió a Pearson como Presidente de la Compañia Brasileña de Tracción en 1915. ${ }^{16}$

Cualesquiera que fueran las actividades de estos empresarios. canadienses, parece que frecuentemente proporcionaban un incremento en la fortuna personal. Sir William Mackenzie da un ejemplo extremo al manifestar "... yo tampoco he estado en São Paulo, pero he sacado un millón de dólares de ahí en los últimos tres aกีos."17

El período más interesante de esta actividad concierne a la Revolución Mexicana, 1911-1928. Siempre me ha extrañado por qué los empresarios canadienses decidieron entrar en México tan tarde en vista de que el Presidente Porfirio Díax, el hombre que había dado treinta años de estabilidad a México, tenía ya más de setenta años. El desconocimiento de la historia mexicana podría ser una de las razones, otra podria ser la confianza expresada por Weetman. Pearson, el dinámico empresario británico, quien aparentemente:

"Charles A, Gauld, The Last Titan, Percival Farquhar American Entrepreneur in Latin America (Stanford, 1964), 30-39, 212-214.

${ }^{18} \mathrm{Ibid} ., 66.77$; F. J. Mulqueen, "A Canadian Enterprise Abroad," Canadian Banker, v. 59 (Winter, 1952), 36-40.

${ }^{17 D}$ D.B. Hanna, Trains of Recollection Drawn from Fifty Years of Railway. Service in Scotland and Canada and told to Arthur Hawhes (Toronto, 1924), 229. 
asumió que todo seguiria igual después de la era de Diaz ${ }^{18}$. 'Si Weetman Pearson no se dio cuenta, ¿cómo podrían verlo los amigos canadienses de F. S. Pearson (ningún parentesco) ? Asi que tenemos, pues, importantes inversiones canadienses en México después del 1900. Inversores canadienses compraron la Compañia de Tranvías de la Ciudad de México, desarrollaxon la Compañía de Luz y Energía de México, y la Compañía de Tranvías, Luz y Energía de Monterrey; construyeron ferrocarriles $y$ plantas de energía ein Chihuahua; y muchos canadienses adquirieron propiedades en áreas muy dispersas para agxicultura o minería. El Banco de Montreal $y$ el Banco Canadiense de Comercio participaron en toda esta actividad. El último abrió una sucursal en la Ciudad de México en 1910, seguro de que "negocios de considerable magnitud estaban asegurados para el banco desde su comienzo"19. En 1913 los bancos ya comenzaban a darse cuenta de lo que significaba mantener una actividad comercial en una situación revolucionaria y tuvieron que hacer frente a los requerimientos de sucesivos gobiernos, frecuentemente de corta duración en el mando, para que compraran bonos. El Banco de Comercio tuvo que comprar bonos del General Huerta en ese año, y en 1930 pidió plañideramente al gobierno canadiense que presentara una demanda de $\$ 1.000 .000$ de dólares más intereses a los mexicanos. Huerta había sido depuesto en 1914 ! O. D. Skelton, el Subsecretario de Estado para Relaciones Exteriores, después de consultar con el Secretario de Asuntos Dominios en Londres, decidió no continuar con la demanda.20

A Luz y Fuerza de México (nacionalizada en 1960) no le fue muy bien durante y después de la revolución: desde 1919 hasta mediados de 1950 no pagó dividendo21. La Compañia de Tranvias de la Ciudad de México estuvo varias veces en manos de los revolucionarios desde 1913 hasta 1916\%2. La Compañía de Tranvias, Luz y Fuerza de Monterrey fue muy poco popular y tuvo dificultades con las distintas fuerzas cambiantes. Sorprendentemente,

${ }^{18}$ Desmond Young, Member for Mexico: A Biography of Weetman Pearson, First I'iscount Cowdray (London, 1966).

${ }^{18}$ Tower a Grey, 18 de marzo de I910, PAC, RG 7, G21/9758-1 (a). El banco recibió las cuentas de "Mexican Light and Power" y "Mexico City Tramways" que anteriormente estaban en el Banco Nacional de México.

${ }^{20}$ Secretary of State for External Affairs (citado en lo succsivo como SSEA) a Secretary of State for Dominion Affairs (SSDA), 29 de marzo de 1930, and O. D. Skelton a SSDA, 23 de junio de 1930, en PAC, RG 7, G21/9758-4.

${ }^{21}$ Gauld, The Last Titan, 57; Miguel S. Wionczek, "Electric Power, The Uneasy Partnership" en Raymond Vernon (cd.), Public Policy and Private Enterprise in Mexico (Cambridge, Mass., 1964), 91.

${ }^{22}$ Información sobre la lucha de Mexico City Tramways durante la revolución está en PAC, RG 7, G21/9758-1 (a) y I (b). 
los directores en Toronto estaban fuera de contacto con la situación y el vice-cónsul en Monterrey declaró:2":

Los problemas de la Compañia parecen surgir del hecho de que está dirigida y controlada en todos sus detalles por un Gerente General y una Junta Directiva en Toronto, quienes desconocen totalmente las condiciones locales y no pueden apreciar la presente situación.

Esta falta de entendimiento de asuntos mexicanos llegó a su culminación clurante el verano de 1916 cuando los directores de la Compañia de Tranvías de Monterrey se unieron con el Banco de Montreal y el Banco de Comercio para pedir ayuda a Washington. Evidentemente no podian tener ningún conocimiento de la situación mexicana pues eligieron el peor momento -las tropas del General Pershing estaban persiguiendo a Pancho Villa en territorio mexicano-. El embajador británico en Washington y el cónsul general británico en México pensaron ambos que este pedido de ayuda era perjudicial para los intereses canadienses.24 Pero este muy bien podría ser el momento en el cual los inversores $y$ empresarios canadienses comenzaron a buscar la influencia y protección de los Estados Unidos en sus relaciones comerciales con América Latina. También indica una decidida falta de conocimiento de la situación comercial durante un proceso revolucionario, especialmente en México donde "la intervención del Gobierno de los Estados Unidos es motivo de indignación..."25

La impresión que deja la misión canadiense de ministros gubernamentales a nueve países latinoamericanos en 1968, y la subsiguiente publicación de Politica Exterior para Canadienses, América Latina, es que Canadá probablemente participará más activamente en la vida de la región. Fácil sería exagerar el alcance de tal participación potencial, ya que América Latina no ha sido, $y$ probablemente no será foco principal de atención para Canadá. Toda participación clel gobierno canadiense en esa región al parecer ha sido 1) reacción ante posibles pérdiclas de mercados tradicionales (los Estados Unidos o Europa, por ejemplo; o 2) el deseo de un gobierno recientemente electo, de imprimir su personalidad en la mente de la población. Ejemplo claro del primer caso son las misiones comerciales de 1866 y' 1941; las iniciativas de

asanford a Spring-Rice, 3 novicmbre de 1915, PAC, RG 7, G21/9758-1 (b) ; Sanford a Hohler, 10 de julio de 1916, G21/9758-2 (a).

s4 Hohler a Grey, 11 de julio de 1916 y Spring-Rice al Gobernador General, 10 de julio de 1916, G21/9758-2 (a).

${ }^{25}$ Ibid. 
los gobiernos de Diefenbaker y Trudeau son ejemplos del segundo26. Pero en ningún caso se ha basado la decisión canadiense en una vehemente necesidad de intervenir más intensamente en la región ${ }^{27}$. Esto puede verse con claridad meridiana en las discusiones que precedieron al envío de las primeras misiones a América Latina.

Canadá, al igual que los demás Dominios Británicos, había alcanzado la igualdad jurídica con Gran Bretaña, la madre patria, en 1926. En la Conferencia Imperial de aquel año, la Declaración Balfour, que fue legalmente confirmada en el Estatuto de Westminster el 11 de diciembre de 1931, estableció la absoluta independencia de cada uno de los Dominios. Y el gobiemo canadiense muy al comienzo determinó expresar dicha independencia abriendo sus propias misiones diplomáticas en Washington, París y To$k^{2}$. Habia al parecer una buena razón para que no fueran más: las misiones resultaban caras y Canadá contaba con recursos limi-

${ }^{20} \mathrm{Tan}$ velozmente pasa $\mathrm{cl}$ tiempo y surgen nuevas crisis en nuestra patria $y$ en cl extranjero, que no parece probable que muchos canadienses se acuerden siquiera de que hubo tal cosa como la misión de 1968. También se puede sugerir que igualmente la han olvidado en los paises que visito. Para comprender mejor las iniciativas del gobiemo de Diefenbaker, véase J. C. M. Ogelsby, The Extent, Focus, and Changes of Canadian Public Interest in Latin America, 1957-1967 (Ottawa, Department of External Affairs, 1967). Picrre Elliot Trudeau venia interesándose en la América Latina desde algunos años antes de su elección. Esto puede observarse en la cantidad de artículos que sobre dicha región había publicado en su periódico crítico, Cité Libre. Puede ser que su inter'śs haya surgido de reconocer que las naciones americanas tiene origen europeo y que todas han tenido que bregar para dominar el ambiente. Puede que también haya influido en 1 l la suposición corriente de que, puesto que los francocanadienses son latinos y católicos romanos, tienen afinidad especial con los pueblos latinoamericanos. Un análisis reciente sobre la validez del concepto de latinidad puede verse en J. C. M. Ogelsby, "French Canada and Latin America", Canadian Forum (Septenber, 1969), 132-195.

${ }^{27 E u g e n e}$ Miller, "Canada and the Pan Amexican Union", International Journal, III (Winter, 1947-48), 24-38; J. C. M. Ogelsby, "Canada and the Pan American Union: 'Twenty Years On", International Journal, XXrv (Summer, 1969), $571-589$.

${ }^{28}$ El proceso evolutivo mediante el cual los paises de la Comunidad, particularmente los lamados "dominios blancos" (Australia, Canadá, Nucva Zelanda, la Unión de Sulaffrica), alcanzaron su independencia, resulta bastante confuso, He hallacio que los latinoamericanos cmplean el modo subjuntivo al referirse a la independencia del Canadá, lo cual muestra que quizá abriguen cicrtas dudas respecto al verdadero alcance de dicha independencia. (La cuestión no se plantea en cuanto al debate actual en Canadá sobre la propiedad estadouniciense de las industrias canadienses, ni sobre la proliferación de académicos estadounidenses en sus universidades, sino que todavía se refiere a la posición del respecto a la Gran Bretaña). Todavía en 1946 cl lider argentino Juan Perón pudo afirmar que existia una diferencia entre Canadá, dominio británico, y la Argentiná, un 
$\operatorname{tados}^{29}$. No obstante, la segunda guerra mundial cambió la perspectiva canadiense al aislarnos de Europa y obligar al gobierno canadiense a buscar substitutos para los mercados perdidos. También comenzó a buscar aliados en el lado americano del Atlántico. ${ }^{30}$

México y Canadá, entre los cuales se dice que existe un problema, según parece no buscaron relaciones mutuas. Pero la presión ejercida por los países del ABC y los Estados Unidos impulsó a Canadá para establecer misiones en Sudamérica y no en países más cercanos a sus fronteras.

Ya en 1938 Brasil había sugerido intercambiar ministro con Canadá. En las etapas iniciales el gobierno brasileño se mostró bastante anuente a que Canadá nombrara un "ministro para el este de Sudamérica", con sede, naturalmente, en Río de Janeiro ${ }^{31}$. El gobierno canadiense del Primer Ministro William Lyon Mackenzie King (el cual era además Ministro de Relaciones Exteriores) no se mostró ansioso de llegar a una decisión. El Departamento de Relaciones Exteriores halló argumentos convincentes para. inclinarse por la Argentina en vez de Brasil, y Skelton, subsecretario de King, confesó que elegir entre páses era dificil, y mucho más en vista de la iniciativa brasileña. Por consiguiente, el gobierno deseaba estudiar más la cuestión. ${ }^{32}$

A principios de 1940 el gobierno argentino empezó también a presentar propuestas, pero King no quiso precipitarse $y$ todavía en agosto se sentía "inclinado a dudar si seria prudente hacer un nombramiento en este preciso momento. América del Sur [escribió] será zona turbulenta mientras dure la guerra"33. Pero de parte de Argentina y Brasil hubo un decidido esfuerzo por llevar al Canadá a una decisión positiva. Según parece, King temía que los gastos de las nuevas misiones fueran demasiado elevados (lo cual ha

estado soberano (La Prensa, 18 de julio de 1946). Evidentemente no hubo ningún oficial de la embajada británica que no aprovechara la oportunidad para enmendar el error, pero la falta de comprensión en torno al Estatuto de Westminster no se limitaba ni se limita a los pueblos británicos. Hay hasta canadienses educados que no parecen comprender muy bien las sutilezas de su constitución.

2a "Legations in Argentina and Brazil, Further Questions" y "Nemorandum for the Prime Minister", 10 de agosto de 1940, Public Archives Record Centre (en lo sucesivo citado como PARG) 282653.

${ }^{30} \mathrm{~F}$. Gordon Skilling, Canadian Representation Abroad, from Agency to Embassy (Toronto, 1945), 249-250; "The Attitude of Canada to the PanAmerican Union", Foreign Research and Press Service, Balliol College; 7 de febrero de 1942 en PARC 283130.

"Wilgress a O. D. Skelton, 7 de febrero de 1938, PARG 282693.

32Skelton a Prime Minister; 16 de febrero y 6 de agosto de 1938, PARG 282693.

${ }^{33} \mathrm{King}$ a Skelton, 13 de agosto de 1940, PARC 282693. 
sido perenne preocupación de todo gobierno canadiense). Sus consejeros le decían que ello se compensaba con el cierre de las misiones europeas. Finalmente, en octubre de 1940 King se decidió y convino en establecer relaciones formales con los dos paises sudamericanos. Pero hubo una demora de casi un año antes de anunciar el nombramiento de los ministros canadienses. ${ }^{34}$

Una de las grandes dificultades que confrontó el servicio exterior del Canadá en 1940-1941 fue la carencia de oficiales de rango $y^{r}$ experiencia suficientes. La guerra ocupaba a mucho de su personal, y según parece, algunos de los oficiales de mayor categoria no consideraban atractivos los puestos en América del Sur ${ }^{35}$. Pero se pudo contar con Jean Désy, ex ministro canadiense en Bélgica y los Países Bajos, el cual estaba muy interesado en estrechar relaciones con América Latina, y se le nombró representante en Río de Janeiro ${ }^{36}$. El nombramiento para la Argentina resultó más difícil.

Hubo varias razones en la tardanza del gobierno canadiense para resolver el nombramiento de su representante en la Argentina. Una fue la escasez de oficiales de mérito, pero más importante fue la presión de Chile en busca de relaciones ${ }^{37}$. El gobierno chileno hizo propuestas mediante su cónsul general en Montreal. Con evidente conciencia de su importancia como la " $\mathrm{C}$ " entre los paises del ABC, el gobierno chileno se sentía postergado. Al mismo tiempo, el cónsul general quería ascender a ministro de su país en Canadá, y asediaba a King, el cual se sentia cada rez más turbado por esa presión. El Primer Ministro canadiense observó que el cónsul general “... quiere un puesto para sí mismo. Tengo por indigno, para no decir algo peor, que presione como lo hace. Habrá que decirle... que por ahora no pensamos ampliar nuestro servicio diplomático en Sudamérica más allá del punto indicado." Las cosas habrían quedado allí a no ser por la intervención del gobierno de los Estados Unidos. ${ }^{38}$

EI ministro estadounidense en Ottawa regresó de una visita a Washington a principios de febrero de 1941. Traía consigo una comunicación del Secretario de Estado de los Estados Unidos, Cor-

34K. H. McCrimmon a H. C. Keenleyside, 18 de junio de 1940, y "Legations in Argentina, and Brazil, Furcher Questions" en PARC 282693; Skelton a Prime Minister, 29 de mayo de 1940, PARC 282658; y SSEA a SSDA, 28 de octubre de 1940, PARC 282693.

- "Skelton a Prime Minister, 24 de noviembre de 1940, PARC 282693.

${ }^{4}$ Wilgress a Glass, 24 de julio de 1941, PAC, RG 20 B 1, v220/29851.

${ }^{37}$ N. A. Robertson (el nuevo subsecretario de Relaciones Exteriores) a Prime Minister, 7 de abril de 1941, PARC 282658.

${ }^{\mathrm{B}}$ Skelton a Prime Minister, 28 de noviembre de 1940 y los comentarios de King, 15 de encro de 194I, PARC 282909. 
dell Hull. Éste expresaba la esperanza de que Canadá otorgara. favorable consideración al intercambio diplomático con Chile. Los Estados Unidos concedian considerable importancia a la estrecha colaboración con Chile "... el cual parecía muy disgustado por haber sido excluido cle la extensión de representación diplomática canadiense en América del Sur."'s9 Sumner Welles, el poderoso subsecretario de estado (1937-1943) añadió su peso unos dias después en nota al gobierno canadiense. Welles consideraba importante para "la defensa hemisférica" que Canadá "satisfaciera la susceptibilidad chilena en esta cuestión, incluyendo a Chile en el plan de representación diplomática en Sudamérica." 40

Mackenzie King, según se ha reconocido desde hace mucho, estaba por lo común dispuesto a seguir los consejos estadounidenses, pero en este caso no se dejó apresurar. En junio, sus consejeros le indicaron la posibilidad de acreditar ante Santiago el ministro canadiense de Buenos Aires. El gobierno podría declarar que procedia así en vista del tradicional concepto del ABC. Y subrayaban que "Ios Estados Unidos han solicitado virtualmente que Canadá lo haga asi:" Calmaron los escrúpulos que King pudiera tener, indicándole que la medida concordaba con las declaraciones de Ogdensburg y Hyde Park relativas a la defensa de las Américas. ${ }^{41}$

Tres meses después, taas recibir la conformidad de Argentina respecto a acreditar un ministro canadiense para Argentina y Chile, el gobierno anunció el nombramiento para dicho puesto, de W. F. A. Turgeon, poderoso sostén del Partido Liberal y' miembro de la Asamblea de Saskatchewan ${ }^{42}$. Los nombramientos de Désy y Turgeon iniciaron la extensión de las relaciones del Canadá con la América Latina, y en 1962 Canadá tenía ya relaciones diplomá-

"Mremorandum de Robertson, 8 de fehrero de 194l, PARC 282909.

"Memorandum a Prime Minister, 15 febrero de 1941, PARC 282909.

"Memorandum a Prime Minister, 7 de junio de 1941, PARC 282909.

WSSEA a SSDA, 25 de julio de 1941 y "Press Release" de 23 setiembre de 1941, PARC 282909. Welles no cejó en su empeño de influir en la política de Canadá respecto a la América Latina. Por el tiempo en que Canadá pensaba nombrar ministro en Chile, èl insistia en que se establecicran relaciones diplomáticas con México. El gobierno canadiense no siguió su consejo esta vez; en noviembre de 1942 nombró un ministro que lo representara en Santiago. Canadá no entabló relaciones diplomáticas con México sino hasta 1944. Sospecho que la influencia de Welles en Ottawa se habia debilitado por la resuelta oposición que junto con el Presidente Roosevelt habia ejercido para mantener al Canadá fuera de la Unión Panamericana. El memorándum de Kecnleyside indicaba un quebran. tamiento en las normalmente buenas relaciones entre Canadá y los Estados Unidos, dado que Welles había afectado gravemente los intereses canadienses mediante argumentos "manifiestamente faltos de solidez" y que él no empleó con terceras partes. Datos relativos al eficaz empeño de los Estados Unidos por 
ticas con todos los países de la región. Ocho años más tarde, poco antes de publicarse Politica Exterior para Canadienses, el gobierno de Trudeau clausuró sus embajadas en República Dominicana, Ecuador y Uruguay. Esto no es preludio de nuevas reducciones, pero ilustra que la política exterior del Canadá en esta región es susceptible de revisión, y que dicha revisión no conduce necesariamente a la expansión. Canadá, con sus intereses mundiales y su dualidad cultural, probablemente enfoque su atención en unas cuantas naciones latinoamericanas en vez de tratar de estrechar relaciones con todas ellas. ${ }^{43}$

En mi opinión, la contribución canadiense en América Latina más interesante ha sido en el campo político. Posiblemente sea de más interés pues proporciona una visión más clara de estos tiempos de incertidumbre. Estoy hablando de la contribución canadiense al movimiento autonomista en Cuba y Puerto Rico, las últimas colonias españolas en América después de las guerras de independencia. Los autonomistas no tuvieron éxito en Cuba, no por falta de esfuerzos, sino porque Cuba eligió soluciones revolucionarias. Los puertorriqueños se han mantenido fieles a la causa autonomista a pesar de los independistas (o separatistas), quienes algunas veces han recurrido a la violencia.

Un interés temprano en la experiencia canadiense es evidente en el artículo de 1837 de José Antonio Saco en el cual expresa su deseo de cambiar el destino de Cuba por el de Canadát4. Irónicamente, la Rebelión de 1837 , que era una protesta contra el poder de la oligarquía, sucedió a la publicación, pero la rebelión no tuvo éxito y lo que asumió importancia para cubanos y puertorriqueños fueron los resultados subsiguientes.

Durante el verano de 1969 , mientras trabajaba sobre este tema

mantener a Canadá alejado de la Unión Panamericana, ver Keenleyside a Prime Minister, 13 de abril 1942, PARC 283130 y Douglas C. Anglin, "The United States Opposition to Canadian Membership in the Pan-American Union: A Canadian View", International Organization, XV (Winter, 1961).

${ }^{+3} \mathrm{El}$ gobierno canadiense ha continuado negíndose a comprometerse plenamaente en la Unión Panamericana. Foreign Policy for Canadians virtualmente sostiene la posición adoptada hace treinta años por el subsecretario de cstado que escribió que Canada estaba preparado para cooperar o participar en aquellos organismos que fueran valiosos para los intereses canadienses, pero "la declaración general que quizá podría formularse es que nos abstenemos cuidadosamente de involucrarnos en todo compromiso político." (Skelton a Wodehousc, 4 de abril de 1940, PARC 265542).

"José Antonio Saco, "Paralelo entre la Isla de Cuba y algunas colonias inglesas escrito por Don José Antonio Saco", Papeles Sobre Guba. (La Habana, 1963), Tomo IIr, 160. 
en la Biblioteca Nacional de Cuba, muchas veces me pareció estar leyendo un texto detallado de historia ctnadiense, tal era lia preocupación de los cubanos con cada paso hacia un "gobierno responsable" y con la Confecleración ${ }^{45}$. En realidad, pareceria que el Canadá era el ideal del político autonomista.40

Los autonomistas cubanos no tuvieron éxito pero aún en los dias sombrios de la Guerra de Independencia de 1895-98 el director cle "El País", uno de varios periódicos autonomistas, dijo en un comentario del Gobicmo de Canadd, de John George Bourinot, que tenía "una importancia excepcional para nosotros en este momento.",it

La intervención estaclounidense inclinó la balanza. Cuba tomó el rumbo seguido por el Dr. Castro actualmente, independiente y relativamente libre sólo después de años de violencia y lucha desde 1898.

En Puerto Rico, los Estados Uniclos intervinieron cuando los puertorriqueños habian alcanzado la autonomia. Habian rechazindo a la revolución, al Grito de Lares, en 1868 y en noviembre de 1897 España les acordó su autononia. En febrero próximo, Luis Muñoz Rivera formó el primer gabinete puertorriqueño. Tres meses más tarcle los Americanos lo invadieron.48

Los pucrtorriqueños se encontraron nuevamente bajo un gobierno imperial $y^{\prime}$ gobernadores designados por Washington astmieron el poder en la nueva colonia. Pero Luis Muñoz Rivera luchó hasta su muerte en 1916 para reconquistar esa autonomía tan fugazmente poseída. No. turo éxito, y es inceresante notar, me purece, qụe su hijo, Luis Muñoz Marin, levantó el estandarte autonomista luego cle un breve galanteo con objetivos socialistas e independislas. Muñoz Marín regresó en 1931 a lo que un gobernador de la isla de la administración de Roosevelt llamó "La Tiema Azotada". Puerto Rico dccayó tanto durante la depresión que no

"Ver por cjemplo La Autonomía Colonial, Colección de Articulos Publicados por 'El Triunfo' Órgano Oficial del Partido Liberal (La Habana, 1887) @ Constitución del Canuld $y$ nolas relatiras a la Confederacion de las provincias Britanicas de la América del Norte (Lá Mabina, 1869).

'D Dr. Antonio de Bustamante y Montoro ha escrito en su ensayo La Ideologia Autonomista (Lil Habana, 1933) que "Este régimen de autonomia colonial está inspirado en las instituciones políticas inglesas y especialmente, por su éxito, en las del Canada."

${ }^{17}$ El Pais, 15 de febrero de 1898.

${ }^{10}$ Ver Lidio Cruz Monclavo, "The Pucrto Rican Political Movement in the 19:h Century," 43; y Robert J. Hunter, "Historical Survey of the Puerto Rico Status Questions, 1898-1965", 52, en Status of Puerto Rico, Selected Bachground Studies prepared for the United States.Puerto Rican Commission on the Status of Puerto Rico (Washington, 1966). 
tuvo más remedio que subir; Muñoz Marín decidió que para poder ayuclar al pueblo de Puerto Rico él tendría que buscar al autogobiemo clentro del firmamento más acaudalado estadounidense antes de perseguir la independencia y la pobrcza. Organizó un programa politico $y^{\prime}$ en 1948 llegó a ser el primer gobernador: elegido de Puerto Rico. En 1952 el pueblo votó abrumadoramente por el golsierno propio dentro del imperio estadouniclense. Puerto Rico estaba ahora completamente en control de sus asuntos internos, pero como un "Estado Libre Asociado" podia contar con fuerte apoyo financiero del gobierno estadounidense para sus proyectos. 40

Los puertorriqueños todavia no habian decidido su posición y en 1967, el 23 de julio para ser exactos, nuevamente fueron a las elecciones para decidir si aprobaban la posición autonomista. (Aquellos quienes disciernen un cierto simbolismo en el mes y año pucden estar seguros de que no fueron elegiclos para commemorar a la contribución canadiense. Fue la fecha mis conveniente para poner en práctica las recomendaciones de la Comisión clesignacla por J. F. Kennedy). Durante años una minoria ruidosa de nacionalistas había recomendado la completa independencia de los Estados Unidos en vista del desarrollo económico que estaba tomanclo lugar en la isla. Los nacionalistas trataron varias veces de dar publiciclad a su posición a principios de 1950 tomando posesión de la "Casa Blanca" puertorriqueña, tratanclo de asesinar al Presidente Farry S. Truman, y provocando un tiroteo en la Casa cle los Representantes de los Estados Unidos. Estos actos no ay'udaron a su causa $y$ nuncal recibieron más del $13 \%$ de los votos en las elecciones puertorriqueñas 50 . In 1967 los puertorriqueños demostraron que la verdaclera elección debía hacerse entre convertirse en estado o mantenerse autónomos. Por una mayoría reducida eligicron nuevamente a la autonomíati. La cuestión no ha sido decidida todavia y puede ser que la próxima vez elegirán entre ser un estado $y$ la independencia.

Lo que a mi me parece importante es que esta isla pequeña en el mar latinoamericano ofrece a los canadienses la oportunidad de verse desde el extranjero. Mario Beaulicu, que fuera Ministro de la Unión Nacional en Quebec, dijo recientemente que su provincia debería ser un Puerto Rico dentro de los Estátos Unidos. El Globe and Mail satirizó su posición, pero Beaulieu, aunque lo sepa o no, no está del toclo desacertado. No sugiero que su

"Thomas Mathews, Luis Muñoz Marin (New York, 1967), 21.18.

${ }^{* 0}$ Huntcr, "The Status Question ...", 117-124.

"San Juan Slar, 24 de julio de 1967. 
plan sea correcto, pero por lo menos vio algo en la posición de Puerto Rico52. En realidad, Puerto Rico tiene mucho en común con Quebec. Ya he hecho hincapié en los elementos separatistas, pero uno no debe olvidarse de que los puertorriqueños son mayormente un pueblo poco violento con una fuerte infiltración anglosajona. Los puertorriqueños generalmente deben ser bilingües para poder adelantar en sus profesiones o negocios. Los anglosajones controlan la mayor parte de los negocios en la isla. La cultura del continente vecino se ha infiltrado en las tradiciones hispánicas y los puertorriqueños deben luchar para mantener esta herencia. Pero por otro lado los puertorriqueños reconocen que su nivel de vida mejorado depende de la participación anglosajona.

Antes de desechar la penetración estadounidense, ellos han, como yo señalara al tratar sobre la cuestión de su posición, elegido. el camino que les proporciona la esperanza de un desarrollo $y$ crecimiento económicos. Tambićn vale la pena notar que la prosperidad creciente ha permitido al gobierno expandir sus programas. culturales y preservar de esta forma la herencia tradicional a pesar: del dinamismo anglosajón.

Si bien la independencia de Cuba puso fin a los aportes canadienses a dicho pais, es interesante observar que las relaciones canadiense-cubanas vienen de muy atrús. Hubo canadienses como voluntarios entre los insurgentes en la Guerra de los Diez Años (1868-1878) ${ }^{63}$; otros, como los capitalistas del CPR y representantes de bancos y cmpresas comerciales canadienses, ingresaron en el país durante la ocupación por los Estados Unidos (1898-1902), y después. Ambos países convinieron en intercambiar misiones diplomáticas en 1945, ya que Cuba, por lo menos en opinión canadiense, estaba al nivel de Chile y México, y un poco por debajo de Argentina y Brasil en orden de importancia. ${ }^{54}$

La actitud de los oficiales canadienses fue en defensa de los intereses financieros clel Canadá durante las luchas revolucionarias contra el dictador Gerardo Machado (1926-1993). Pero la reciente revolución de Fidel Castro brindó al gobierno canadiense del Primer Ministro John Diefenbaker (1957-1963) y a los sucesores suyos la oportunidad de acentuar la creciente determinación de mos-

"Toronto Globe d Mail, 13 de agosto de 1970.

${ }^{5}$ Vaillancourt a SSEA, I8 de julio de 1946, PAC, MG 30 a 11.

"El Banco Mercantil del Canadá (en 1901 pasó a ser 'The Royal Bank of Canada) se estableció en Cuba en 1898; pronto compró el Banco de Oriente y el Banco del Comercio. Esta operación inicial en el Caribe creció hasta abarcar más de cincuenta en dicha región. Respecto al orden de importancia de los. respectivos países, ver "Miemorandum for the Prime Minister", 10 de agosto. de 1940 , PARG 282693. 
trarse más independiente respecto a los rumbos que en política internacional marcaban los Estaclos Unidos. ${ }^{55}$

Canadá, en la época posterior a Mackenzie King (aunque éste se retiró en 1948, sus representantes continuaron políticamente activos hasta que fueron derrotados en 1957$)$, se ha venido apartando cada vez más de la relación norteamericano-canadiense caracterizada por la llamada "diplomacia silenciosa"56. Ello significaba que ambas naciones por lo común resolvían sus problemas mutuos rentre bambalinas. Pero a partir de 1956 los gobiernos canadienses se han inclinado a manifestar un nacionalismo creciente y una conciencia cada día mayor de que tal vez los canadienses no fueran ni británicos ni estadounidenses. Diefenbaker, igual que su ídolo Sir John A. Macdonald, el primero que ocupara en Canaclá el cargo de Primer Ministro, era un nacionalista canadiense, y si bien procuraba invocar la tradición británica como antídoto contra la influencia de los. Estados Unidos, sus actos eran manifiestamente independientes ${ }^{57}$. Así las cosas, la creciente brecha entre Castro y el gobierno de los Estados Unidos permitió a Diefenbaker destacar la posición canadiense. Pocos eran quienes pensaban que Canadá iba a salir ganando en lo económico mediante el continuado reconocimiento del gobierno castrista, pero muchos comprendieron las ventajas políticas que ello encerraba. ${ }^{58}$

El no romper relaciones con Castro por las medidas cubanas

suskelton a Wrong, 20 de agosto de 1931, PARG 265367. Como subsecretario de Relaciones Exteriores, Skelton sostenía que "Con base en principios generales yo me inclinaria a simpatizar bastante con el movimiento revolucionario. Pero al presente no puede el mundo soportar muchas perturbaciones más, y. de haber cualquier posibilidad de ataque contra los bancos canadienses, supongo que habríamos de considerar la continuada dictadura del Presidente Machado como 'el mal menor:"

"Una evaluación de dicha técnica puede verse en "Principles for Partnership" por Livingston Merchant y A. D. P. Heeney. Y ver tambièn John Saywell (ed.) Canadian Annual Review 1965 (Toronto, 1966), 259-262.

${ }^{57} \mathrm{Al}$ parecer, a Diefenbaker no se to estimaba mucho en Washington, ya que su posición respecto a Cuba y el no haber procedido decisivamente en el convenio sobre armas nucleares con los Estados Unidos, no le acarrearon simpatía. El Pxesidente Kennedy no confiaba en él (por ejemplo ver Theodore C. Sorenson, Kennedy (New York, 1965), 575-576 o Arthur Schlesinger, A Thousand Days, John F. Kennedy in the White House (Cambridge, Mass., 1965), 313. John W. Holmes ha escrito un ensayo muy pensativo sobre "Nationalism in Canadian Foreign Policy" en Peter Russell (ed.), Nationalism in Canada (Toronto, 1966), 203-220.

${ }^{88}$ Kenncth McNaught, "Castro's Cuba, Ottawa, Washington," Saturday Night, v 76, no. 2 (January 21, 1961), 7-11; Toronto Telegram, 5 January, 1961; Toronto Globe \& Mail, 5 January 1961; Le Devoir, 21 avril 1961; Toronto Star, 6 January 1961; Le Soleil, 25 octubre 1962; Le Devoir, 5 février 1962; François Piazza, "Punta del Este: La Farce est Jouée", Cité Libre, XIII (mars, 1962), I8-19. 
de nacionalización que afectaron a bancos y otras empresas canadienses, ni con ocasión de la crisis de los cohetes cubanos en 1962, que puclo parecer amenazante a Canadá, simplemente puso de relieve el hecho de que el señor Diefenbaker queria seguir su.propio clerrotero. Lester Pearson, jefe del partido. liberal que habia sido oficial del servicio de relaciones exteriores y.ministro en el Departamento de Relaciones Exteriores en la época de Mackenzie King, fue el sucesor de Diefenbaker en 1963. Su gobierno mostró cierta ambivalencia respecto a relaciones con los Estados Unidos. Pearson habia participado íntimamente en el sistema de la "diplomacia silenciosa", pero aun él se sintió frustrado por causa de la guerra de Vietnam, $y$ eligió la ciuclad norteamericana de Filadelfia como sitio en donde hacer una cleclaración pública sobre la politica de bombardeos del presidente Johnson ${ }^{50}$. Al retirarse Pearson, Trudeau se convirtió en jefe del partido y Primer Ministro. Era ajeno a la antigua politica externa liberal; $y$ al igual que Dielenbaker, resolvió proseguir un rumbo más canadiense en cuanto i relaciones exteriores.

El futuro de las relaciones canadiense-latinoamericanas continuará aparentemente siguiendo las lineas bosquejadas en este trabajo. A sabiendas o no (yo creo que no) la política exterior del. gobierno de Trudeau refleja la continuidad de las relaciones canadiense-latinoamericanas clesde 1867. El comercio, las inversiones, comunicaciones, la educación (por intermedio de los esfuerzos de; los misioneros), y el desarrollo han sido por mucho tiempo objelos de mayor interés en el contacto canadiense con América.Lalina. Pero el gobiemo actual ¿realizará mucho más de lo que: se realizó antes? Sospecho que no. Después de toda la publicidad. acordada a la misión del gobierno de 1968, que fue motivo de entilsiasmo entre los interesados en. el Canadá.y los nueve paises visitados, el informe presentado muestra cuán modestas eran las intenciones del gobierno. En realidad, el único aumento en el interés del gobierno que nosotros hemos visto o notado, fue en la suma adicional de veinte millones de dólares para fondos de clesarrollo asignados a proyectos latinoamericanos (especificamente en América Central, Colombia, Brasil y Perú) ${ }^{60}$ : Esto es además de los diez millones destinados anualmente al Banco Interamericano de Desarrollo. Aun con esc aumento, menos del diez por ciento del presupuesto actual de lat Agencia Internacional Canadiense de Desarrollo esti destinado para América Latina.

¿Fay más esperanzas en otros sectores de actividad guberna-

Canadian Almual Revietu, 1965, 221-223.

"Toronto Globe \& Mail, 13 Angust 1970. 
mental? Creo que industria y comercio continuarán tratando de persuadir a los hombres de negocios canadienses a ser más competitivos, pero sin mucho éxito. Como notara John Flarbron hace algunos años, los hombres de negocios canadienses prefieren no embarcarse en nuevas empresas si sus negocios en el mercado estadounidense o canadiense están prosperando ${ }^{3 x}$. El Departamento de Relaciones Exteriores, que extendió su interés en América Latina hacc dicz años, ya ha sufrido reducciones en su personal en Ottawa y América Latina. No estoy en desacuerdo con la decisión búsica cle cerrar ciertas cmbajadas en el exterior, pues clespués de todo la representación internacional canadiense es mas grande que la de cualquier país de población comparable, y no creo necesario que estemos representados en todo país de América Latina. Pero me preocupan las reducciones en el personal de la División de América Latina. Aquellos de nosotros quienes pensamos que podria haber un aumento en el intercambio intelectual y cultural, por más pequeño que fuera, tendremos muchas esperanzas de verlo realizarse. La División de América Latina tendrá poca oportunidad de incluir estos asuntos en un programa ya sobrecargado.

El eterno problema de la participación politica en el sistema interamericano continuará siendo eso: un eterno problema. Francamente, el gobiemo sabe que hay demasiados canadienses que se oponen al ingreso de Canadá en la OEA ${ }^{122}$. Sin embargo, irá tán lejos como pueda sin asumir la plena condición de miembro de dicho organismo. ${ }^{\text {:3 }}$

Toda futura expansión de las relaciones entre Canadá y América Latina habrá de basarse en un conglomerado de canadienses mejor informados. Se ha producido por cierto una notable expansión en la enseñanza de asuntos latinoamericanos en las universidades durante los últimos siete años ${ }^{0.4}$. Ya comenzamos a ver el fruto de esa expansión, en el número de jóvenes canadienses que se han entregado a estudios sobre América Latina. Conforme estos jovenes así instruidos penetren en las profesiones, el comercio, la politica y la educación, observaremos que Américal Latina y cada una

${ }^{01} J o h n$ Harbron, "Canadian Exporters, Investors can find bright prospects in growing Latin Amcrican market", Canadian Business, v. 33 (October, 1960), 51-60. "Ogelsby, Canadian Public Interest.

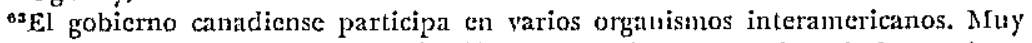
recientemente ingresó en la Organización Panamericana para la Salud y está en proceso de ingresar en el Banco Interamericano de Desarrollo. El 2 de febrero de 1972 Canadá fuc admitido como observador en el sistena interamericano.

"J. C. M. Ogelsby, "Latin Amcrican Studies in Canada", Latin American Research Review, II (Fall, 1966), 80-88. Hernan Konrad, profesor en la universidad de Calgary, tienc el resultado de su reciente investigación, la cual itustra hasta dónde han cambiado las cosas desde 1966. 
de las partes que la integran, recibirán mayor atención en Canadá. Quizá esto produzca en los latinoamericanos un reciproco interés. Pero hay algo que parece cierto: que de ahora en adelante las relaciones de Canadá con la América Latina ya no serán tan anodinas oomo en el pasado. 